\title{
Macbeth sous les bombes, Shakespeare sous la neige : un souvenir d'enfance d'Edward Bond
}

David Tuaillon

\section{(2) OpenEdition}

\section{Journals}

\section{Édition électronique}

URL : http://journals.openedition.org/shakespeare/1947

DOI : 10.4000/shakespeare.1947

ISSN : 2271-6424

Éditeur

Société Française Shakespeare

\section{Édition imprimée}

Date de publication : 1 avril 2013

Pagination : 149-166

ISBN : 2-9521475-9-0

\section{Référence électronique}

David Tuaillon, «Macbeth sous les bombes, Shakespeare sous la neige : un souvenir d'enfance

d'Edward Bond », Actes des congrès de la Société française Shakespeare [En ligne], 30 | 2013, mis en ligne le 03 avril 2013, consulté le 03 mai 2019. URL : http://journals.openedition.org/ shakespeare/1947; DOI : 10.4000/shakespeare.1947 


\section{Shakespeare et la mémoire}

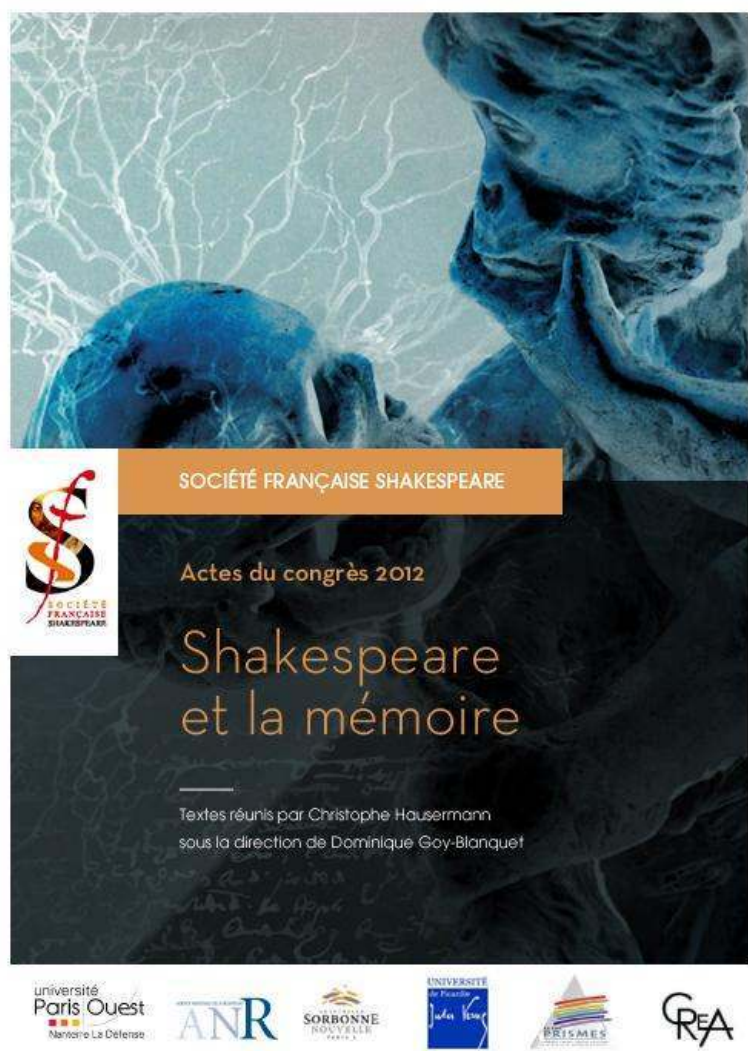

actes du Congrès

organisé par la

SOCIÉTÉ FRANÇAISE SHAKESPEARE

les 22, 23 et 24 mars 2012

textes réunis par

Christophe HAUSERMANN

sous la direction de

Dominique GoY-BLANQUET 


\section{COUVERTURE}

d'après l'affiche de Claire Colombet

conception graphique et logo

Pierre Kapitaniak

mise en page et corrections

Christophe Hausermann

(C) 2012 Société Française Shakespeare

Institut du Monde Anglophone

Université de Paris III - Sorbonne Nouvelle

5 rue de l'École de Médecine 75006 Paris

www.societefrancaiseshakespeare.org

Tous droits de traduction, de reproduction et d'adaptation réservés pour tous les pays 


\title{
MACBETH SOUS LES BOMBES, SHAKESPEARE SOUS LA NEIGE : UN SOUVENIR D'ENFANCE D'EDWARD BOND
}

\author{
David TUAILlON
}

\begin{abstract}
Nous nous proposons d'aborder l'idée de mémoire de Shakespeare très littéralement, mais à rebours, en tant que "mythe personnel », pour reprendre la notion suggérée par Gisèle Venet, mais également en tant qu'éclairage du mythe personnel d'un auteur contemporain, en l'occurrence, le dramaturge Edward Bond.
\end{abstract}

We will deal with the idea of Shakespeare's memory literally, but in retrospect, as a "personal myth", referring to Gisèle Venet's notion, and also as the insight on a contemporary author's personal myth, namely Edward Bond.

$\mathrm{C}^{\mathrm{s}}$

serait une banalité d'affirmer que, comme tous les auteurs britanniques, Bond entretient un rapport très fort et très personnel avec l'œuvre et la figure de Shakespeare. Une étude intertextuelle trouverait beaucoup à dire sur l'innervation de l'œuvre de Bond par celle de Shakespeare (à commencer par sa réécriture de Lear de $1971^{1}$ ). Nous souhaiterions ici plutôt, afin de cerner la singularité de ce rapport qui tient justement à un acte de mémoire, observer et rapprocher deux expériences de théâtre de natures différentes qui lient Edward Bond à William Shakespeare.

La première de ces expériences est la rencontre même de Bond avec le théâtre, à l'adolescence - du moins telle qu'il la raconte luimême $^{2}$. Cette rencontre n'avait rien d'acquis. Bond est en effet issu d'un milieu extrêmement modeste qu'il décrit comme « lower working class 3 ». Ses parents, d'origine paysanne, étaient analphabètes et son

\footnotetext{
${ }^{1}$ Lear, in Edward Bond, Plays 2, Londres, Methuen, 1978 ; création : mise en scène de William Gaskill, Royal Court Theatre Londres, 29 septembre 1971. En ce qui concerne cette dernière pièce, le conditionnel n'est pas de mise puisque la comparaison avec l'original est la lecture la plus classique, voir l'excellente synthèse de Patricia Hern dans la «Student Edition » de la pièce (Londres, Methuen Drama, 1983, p. xxvi-xliii).

${ }^{2}$ Notamment dans « Drama and the Dialectic of Violence », entretien avec A. Arnold, in Theatre Quarterly, vol.2.5, 1972, p. 4-12, dans le chapitre « Theatre-in-Education (TiE) » in Edward Bond, Letters, selected and edited by Ian Stuart, vol. 4, Amsterdam, Harwood Academic Publishers, 1998, p. 105-138 (partiellement publié en français sous le titre « Du théâtre à l'école », 1993-1995, p. 11-19, trad. : D. Tuaillon et M. Vittoz, montage : L. Hémain in Lexi/textes 7, 2003-2004, Paris, L'Arche/ Théâtre National de la Colline, 2003) et dans le premier chapitre de ses Entretiens avec David Tuaillon, "S'il y a une chose que Shakespeare ne fait pas, c'est désespérer », Paris, Archimbaud-Les Belles Lettres, 2013.

3 « Drama and the Dialectics of Violence », op. cit., p. 4.
} 
entourage se méfiait du savoir et de la culture, qui restaient considérés comme la propriété de la bourgeoisie et leur demeuraient inaccessibles. Bond a d'ailleurs fort peu fréquenté l'école: après une longue interruption due à la guerre, il fut jugé incapable de passer l'examen eleven-plus qui lui aurait donné accès à la grammar school et il végéta à la secondary modern school de Crouch End en attendant ses quinze ans, l'âge légal pour sortir du système éducatif. Bond s'est ainsi construit, y compris comme écrivain, en autodidacte et par conséquent sa relation personnelle à Shakespeare s'est établie en dehors de la lecture officielle imposée aux écoliers britanniques et du lien culturel normalisé par l'éducation.

Cette rencontre eut lieu vraisemblablement en 1949, lorsque Bond assista, avec sa classe, à une représentation de Macbeth au Bedford Theatre (aujourd'hui disparu) sur Camden High Street par Donald Wolfit, alors dans la pleine maturité de son art et au sommet de sa notoriété - en particulier grâce aux lunch performances, abrégés de pièces de Shakespeare qu'il avait donnés pendant le Blitz. Le petit Bond ne connaissait du monde du spectacle que le music-hall, où travaillait une de ses sœurs - elle était l'assistante d'un magicien et se faisait scier en deux ${ }^{4}$. On ne se hasardera pas ici à spéculer sur l'influence de tels spectacles sur l'imaginaire d'un petit garçon qui allait devenir un auteur réputé pour sa violence - il y aurait, là encore, certainement matière pour de riches recherches sur la mémoire du music-hall dans l'œuvre de Bond 5 .

Cette représentation de Macbeth fut pour l'adolescent (du moins, tel que l'adulte le relate) bien autre chose qu'une révélation esthétique ou un rituel culturel. Devant ce qui est probablement l'une des pièces les plus fantastiques de Shakespeare, que tout enfant voit sans doute avec la même irréalité qu'un conte de fée, Bond dit avoir au contraire éprouvé « a feeling of total recognition ${ }^{6}$. » Aussi surprenant que cela puisse paraître, ce que Bond « reconnaît » là est l'expérience qui avait été la sienne peu d'années auparavant, celle d'un enfant à Londres sous les bombes et les missiles allemands. Plus tard, il put affirmer : «I knew all these people, they were there in the streets or in

\footnotetext{
4 Bond, cité in Malcolm Hay \& Philip Robert, Bond a Study of his Plays, Londres, Eyre Methuen, Modern Theatre Series, 1980, p. 17

5 Lui-même y a un peu réfléchi, voir Tony Coult, The Plays of Edward Bond, Londres, Eyre Methuen Theatre File, 1979, p. 13 et 85.

6 « Drama and the Dialectics of Violence », op. cit., p. 4-5.
} 
the newspapers - this in fact was my world 7. " Il dit avoir alors reconnu dans les sorcières, ces " secret, black, and midnight hags ${ }^{8}$ ", les aviateurs allemands qui avaient essayé de le tuer durant quatre ans et vit dans le personnage de Macbeth, un homme qui, lui aussi, tue des enfants, l'incarnation de la figure diabolique de Hitler, telle qu'elle était dépeinte par la propagande de la BBC. Sur la scène, il voyait exposées ses propres interrogations sur un mode absolument concret, alors que personne autour de lui, dans son entourage social ou familial n'avait mis de mots sur ce qu'il avait vécu :

For the very first time in my life - I remember this quite distinctly - I met somebody who was actually talking about the life I've been living, the political society around me. Nobody else had said anything to me about my life at all ever 9 .

Cette représentation de Macbeth donnait tout simplement une perspective de sens à sa propre vie que rien ni personne d'autre ne lui avait donnée jusqu'alors. On reconnaît certes là un processus cathartique assez classique d'une expérience traumatique refoulée, mais on y lit surtout une authentique «scène primitive » dont la mémoire (aussi idéalisée ou postérieurement reconstruite soit-elle), relue et réécrite, fonde une existence et une vocation - et, dans le cas qui nous occupe, invente un écrivain et un projet d'écriture. Bond a accrédité explicitement l'influence de cette représentation sur sa décision postérieure d'écrire pour la scène ${ }^{10}$, et il est, de fait, très frappant de lire dans l'interprétation qu'il avance de ses effets sur lui, des lignes de forces qui seront celles de l'ensemble de son projet dramatique : la reconnaissance du théâtre comme la possibilité d'un discours, ou au moins d'une représentation, de la part du réel que l'expérience immédiate ne parvient pas à saisir; une perception instinctive de la fonction métaphorique active du théâtre, qui ne relève pas de l'onirisme ou du symbolisme mais qui propose un éclaircissement direct de l'expérience par l'imagination; enfin, la lecture de l'acte de représentation moins par le mimétisme du réel que comme fiction sur le réel, et de sa signification moins comme une analyse que comme une perspective ouvrant le présent à des

\footnotetext{
7 Ibid.

8 Macbeth, IV.i.46.

9 "Drama and the Dialectics of Violence », op. cit., p. 5 .

${ }^{10}$ Ibid., p. 6.
} 
significations plus profondes.

Lectures à rebours ou réelles intuitions ? C'est en tout cas dans cette expérience première et cette mémoire, dont il fit de Shakespeare le responsable, que Bond lit l'origine de sa propre vocation théâtrale. $\mathrm{Et}$, si «mythique » soit ce récit, on ne peut s'empêcher d'y déceler, venant de Bond, plus que la reconnaissance d'une influence, la conscience d'un relai, d'auteur à auteur, à quelque trois siècles et demi de distance, qui fonde en grande partie son œuvre future.

Parmi ce que Bond dit recevoir de Shakespeare avec Macbeth, se trouve aussi l'idée d'une approche morale, selon une vision, elle aussi, personnelle. Bond lit et salue le personnage de Macbeth non comme personnification $\mathrm{du}$ mal comme faute, finalement et heureusement condamnée, mais comme la personnification du mal accepté comme un fait. Il le considère, parmi les personnages de la pièce, comme celui qui a le plus d' « insight » et en fin de compte le plus $d^{\prime}$ " humanité ${ }^{11}$ ", parce qu'il regarde en face ses actes, sans se dissimuler derrière les déplorations ou les excuses courantes qui autorisent à oblitérer le sens moral ou à le convoquer après coup et ainsi justifier toutes les violences - une rhétorique qu'il dut sans doute beaucoup entendre durant la guerre. Au contraire: ce que Bond considère comme une absence de jugement permet à Macbeth de dire ce qu'il fait en pleine lucidité, d'aller au terme de ses logiques et ainsi de témoigner ès qualités - fussent les pires - de ce qu'il est.

En suivant cette lecture, on constate en effet que si, dans la pièce, la dissimulation et l'hypocrisie font partie intégrante des vices délibérés et notoires de Macbeth ${ }^{12}$ ses soliloques témoignent au contraire de la plus entière lucidité sur ses actes et sur lui-même. Il parvient en effet toujours à décrire ses actions et à leur donner un sens, en témoignant du mouvement de sa pensée et de l'imaginaire qui les accompagne et en les inscrivant dans l'ordre du monde autour de lui : depuis l'approche du meurtre, devant sa vision du poignard qui lui montre le chemin,

It is the bloody business which informs

Thus to my eyes. Now o'er the one half-world

Nature seems dead [...]

\footnotetext{
${ }^{11}$ Entretiens avec David Tuaillon, op. cit., chap. 1.

12 « МАСВЕТн. [...] and makes our faces / Vizards to our hearts, disguising what they are. » et Macduff le qualifie, entre autre, de « false, deceitful », III.ii.34-35 et iv.iii.58.
} 
I go, and it is done. The bell invites me. (II.i.48-50, 72)

à la première prise de conscience, implacable, à la découverte de l'assassinat de Duncan :

There is nothing serious in mortality.

All is but toys. Renown and grace is dead,

The wine of life is drawn, and the mere lees

Is left this vault to brag of

de sa détermination au pire,

All causes shall give way, I am in blood

Stepp'd in so far, that should I wade no more

Returning were as tedious as go o'er.

Strange things I have in head that will to hand,

Which must be acted ere ther may be scann'd

(III.iv.137-141)

jusqu'à l'ultime constat de sa corruption :

I have almost forgot the taste of fears.

The time has been my senses would have cool'd

To hear a night-shriek, and my fell of hair

Would at a dismal treatise rouse, and stir

As life were isn't. I have supp'd full with horrors,

Direness familiar to my slaughterous thoughts

Cannot once start me. Wherefore was that cry?

(v.v. 9-15)

Macbeth ne se ment jamais à lui-même (ou aux spectateurs ?) : il dit toujours ce qu'il fait et ce que fait de lui ce qu'il fait, si bien que Bond lui accorde, en fin de parcours, « une curieuse forme d'innocence ${ }^{13}$ » - c'est-à-dire (pour éclaircir ce terme dont Bond fait un usage fréquent et très personnel) une conscience intègre, fondamentalement et radicalement humaine, de ses actes, dont ne saurait se prévaloir aucun des princes justiciers, en guerre contre ses vices et sa tyrannie au nom de la vertu des rois, ceux qui, comme Macduff, sont si prompts à se lamenter du mal ${ }^{14}$, mais que seul le désir de vengeance personnelle pousse réellement à agir ${ }^{15}$. Au bord de sa

13 Entretiens avec David Tuaillon, chap. 1.

14 MACDUFF. «O horror, horror, horror » (II.iii.62) et «Bleed, bleed, poor country!» (IV.iii.32) ou « O Scotland, Scotland ! (IV.iii.100) ou encore: "O relation / Too nice and yet too true! » (IV.iii.173-174)

15 Macbeth, IV.iii: c'est seulement dans les derniers vers (232-236), récit de l'assassinat de ses enfants et de Lady Macduff, que Macduff aiguillonné par Malcolm, se décide à prendre les armes contre Macbeth, alors qu'ils ont consacré toute cette très longue scène à le conspuer. 
chute, Macbeth peut ainsi faire preuve d'une soudaine sagesse lors de son célèbre envoi en forme de vanité méditative, appréhender l'éternité des «Tomorrow, and tomorrow, and tomorrow» et finalement énoncer le (non-)sens de la vie tout entière comme « a tale / Told by an idiot, full of sound and fury / Signifying nothing ${ }^{16}$ ».

Cette innocence se reconnaît aussi dans le cauchemar de Lady Macbeth, avec la persistance de cette question que son imagination fait resurgir dès que la conscience endormie lui laisse le champ libre :

Yet here's a spot [...] What need we fear who knows it, when none can call our power to account? Yet who would have thought the old man to have had so much blood in him. (v.i.19-28)

Question insistante, élémentaire, désemparée, incroyablement simple, presque infantile et tellement humaine, qui résiste au cynisme et à la corruption, à la souillure du meurtre et à toute intelligence - si bien que la tache que Lady Macbeth tente en vain d'effacer dans son sommeil pourrait bien être non la marque indélébile de son crime mais cet ultime résidu d'innocence irréductible qui l'oblige à savoir ce qu'elle a fait, et ces «parfums d'Arabie », par lesquels elle sait ne jamais pouvoir purifier sa main inflexible de meurtrière (mais qui reste à ses yeux endormis « cette petite main ${ }^{17}$ »), seraient alors l'image d'un désir à jamais inaccessible de retrouver une innocence pleine.

Apparaît alors, devant le génie du mal, un Macbeth qui se connaît lui-même en connaissant ses actes ${ }^{18}$. On est même tenté de lire dans sa soudaine crainte de ne plus jamais pouvoir dormir, parce qu'avec Duncan, il aurait tué le sommeil ${ }^{19}$, l'annonce métaphorique de cette conscience née du premier meurtre. C'est là donc que réside, paradoxalement, la portée morale du personnage et avec lui du geste de l'écrivain. Bond prête en effet à la tragédie la vertu - qui ne relève en rien de la catharsis aristotélicienne -, de permettre l'observation lucide du mal afin de le mesurer et de le comprendre dans les termes de la situation qui le produit. S'il voit donc en premier lieu dans ce

\footnotetext{
16 Macbeth, v.v.19-28.

17 « Here's the smell of the blood still : all the perfumes of Arabia will not sweeten this little hand. Oh, oh, oh! » Macbeth, v.i.34-35.

18 C'est ce qu'implique finalement, et malgré sa tonalité de regret, son vers après le meurtre du roi « To know my deed, 'twere best not know myself. » Macbeth, II.ii.73.

19 « Methought I heard a voice cry 'Sleep no more, / Macbeth does murder sleep', the innocent sleep [...] Still it cried sleep no more [...] Macbeth shall sleep no more ", Macbeth, II.iii.35-36 et 41-43.
} 
personnage un salutaire porteur de vérité, il en attribue surtout le mérite à Shakespeare et célèbre ainsi sa capacité à connaître et à dire cette vérité comme une grande qualité morale. C'est enfin là, plus que dans toute volonté de jugement, que Bond situe la vraie mission du théâtre - qu'il fera sienne : « Saints have nothing interesting to say to us. But Macbeths do ${ }^{20}$. »

Ceci permet, en retour, à Bond d'opérer une lecture très matérialiste de la pièce. Macbeth n'est pas pour lui une pièce sur les forces surnaturelles et la chute des âmes dans le mal, selon un point de vue téléologique, mais un discours sur la condition humaine dans ses paradoxes. Il y voit des personnages moins manipulés par des puissances qui leur échappent que concrètement aux prises avec les conséquences de leurs choix et de leurs actions et tentant de répondre aux situations qui en découlent ${ }^{21}$. Il rejoint ainsi en partie la lecture de Gisèle Venet, qui voit dans la pièce une «tragédie du libre arbitre » où Macbeth, dans ses soliloques, témoigne d' " un libre arbitre qui librement s'aliène 22 » à ceci près que c'est pour lui la conscience qui prédomine sur la liberté ${ }^{23}$. Cette hypothèse permettrait peut-être de trancher le dilemme interprétatif au sujet de son acception finale de la mort, entre un «aveuglement volontaire» ou un « recours à la liberté 24 », en le comprenant comme l'acceptation de la responsabilité de ses actes jusque dans ses ultimes conséquences ${ }^{25}$, à la manière de Richard III qui s'élance dans son duel fatal en s'écriant : "I have set my life upon a cast, / And I will stand the hazard of the die ${ }^{26}$. »

Durant toute sa vie d'écrivain, Bond a reconnu Shakespeare

\footnotetext{
${ }^{20}$ Entretiens avec David Tuaillon, chap. 1.

${ }^{21}$ Ibid.

22 Gisèle Venet, Temps et vision tragique, Shakespeare et ses contemporains, Paris, Presse Sorbonne Nouvelle, nouvelle édition, 2002, p. 154 et p. 149.

23 Malcom Evans a émis une telle idée d'un Macbeth poussé par une volonté de savoir, à partir d'une opposition de deux discours constitutifs de la pièce, celui de la loi naturelle et divine, d'une part, porté par les chevaliers contre celui du couple Macbeth et celui des sorcières, d'autre part, chargé des verbes « know " et "see " et qui s'achève sur la signification par "rien ", comme un appel à décrire perpétuellement ("The 'nothing' signified [...] calls perpetually to account. »), Malcolm Evans, "Signifying Nothing », in John Wain, éd., Shakespeare: Macbeth, a Casebook, Londres, Macmillan, 1968, p. 263280 .

24 Venet, op. cit., p. 154 .

25 Germaine Greer a déjà avancé, mais dans une perspective chrétienne, l'idée d'un Macbeth assumant ses péchés jusque dans son juste châtiment: Germaine Greer, « Macbeth: Sin and the Action of Grace », in Wain, op. cit., p. 263-270.

${ }^{26}$ The Tragedy of Richard the Third, v.v.9-10.
} 
comme un référent dominant pour sa pensée du théâtre - et nous pouvons personnellement témoigner de la fréquence des allusions qu'il y fit durant la série d'entretiens qu'il nous accorda l'an passé. Il le mentionne toujours comme jalon moral, fréquemment à travers trois personnages, dépositaires selon lui d'un aspect singulier d'une interrogation et d'une expérience fondamentale de l'humanité: Hamlet, l'intellectuel, Lear, l'inquisiteur radical, et, naturellement, Macbeth l'extrémiste - dans le sens (lui aussi très personnel) d'un personnage qui va à l'extrême et en assume le coût. La dernière réplique de Lear, au seuil de la mort « Pray you, undo this button ${ }^{27}$ », dans laquelle Bond voit l'agencement du grand et du petit, du monde et de l'humain, de l'ordre et du désordre, est pour lui une source de méditation inépuisable - au point qu'il y a même directement répondu dans une de ses dernières pièces, People, où un personnage meurt en répétant obsessionnellement, pas moins de vingt-huit fois : « Do your button up ! 28 »

Il n'est d'ailleurs pas rare que les personnages des pièces de Bond soient de nouveaux avatars de ces trois personnages ou leur répondent: en dehors de sa réécriture de King Lear, dont le personnage éponyme a gardé jusqu'au nom, il imagina Hécube dans The Woman ${ }^{29}$ comme une « female Lear ${ }^{30}$ » et plus récemment il nous confiait avoir baptisé le personnage principal du Crime of the Twenty First Century ${ }^{31}$, Sweden, en clin d'œil (aussi facétieux que géographiquement imprécis) au prince d'Elseneur ${ }^{32}$. Cette dernière pièce s'achève d'ailleurs sur une glaçante image de la condition humaine contemporaine, qui matérialise directement l'imprécation du roi Lear au-dessus du corps de Cordelia : « Howl, howl, howl, howl ! O, you are men of stone $33 »$. On y voit un personnage dans un cachot gris aseptisé, debout à côté d'une pierre carrée, comme une statue tombée

\footnotetext{
${ }^{27}$ King Lear, v.iii.284.

28 People, in Edward Bond, Plays 8, Londres, Methuen, 2006, p. 108.

29 In Edward Bond, Plays 3, Londres, Methuen, 1987 ; création : mise en scène de l'auteur, National Theatre (Olivier Stage), Londres, 10 août 1978.

30 Entretien à The Observer 15/01/78, cité in Hay \& Roberts, op. cit. p. 240.

${ }^{31}$ The Crime of the Twenty First Century, in Edward Bond, Plays 7, Londres, Methuen, 2003 ; création : mise en scène de Leander Haussman, Schauspielhaus, Bochum, 28 mai 1999 .

${ }^{32}$ Entretiens avec David Tuaillon, chap. 8: «La réalité ne devient praticable que lorsqu'elle vous a dit sa signification ».

33 King Lear, v.iii.232.
} 
incapable de remonter sur son socle, et qui «hurle», non pas seulement quatre, mais sept fois 34 .

Toutefois, afin de suivre le travail de mémoire de cette rencontre avec Shakespeare, nous allons plutôt nous arrêter sur cette pièce crépusculaire et curieusement contemplative, écrite en 1973, Bingo ${ }^{35}$, qui s'autorise à mettre en scène le personnage de William Shakespeare lui-même.

Créée avec Bob Peck dans le rôle principal, reprise ensuite par la Royal Shakespeare Company avec John Gielgud, devenant ainsi la première pièce contemporaine jouée au Swan ${ }^{36}$, Bingo reste une des pièces de Bond les plus jouées en Grande Bretagne - une production du Festival de Chichester a d'ailleurs été reprise cette année à Londres, au Young Vic, avec Patrick Stewart et obtenu un certain écho 37. La pièce est en revanche méconnue en France: traduite par Jérôme Hankins pour Alain Milianti qui l'a créée au Festival d'Avignon en $1994^{38}$, elle n'a pas connu de mise en scène significative depuis. Signalons également (sans nous attarder afin d'éviter de nous engouffrer dans une tout autre problématique) que Bingo s'articule avec une seconde pièce, The Fool, consacrée au poète paysan John Clare 39.

La pièce exploite diverses anecdotes (et ragots) de la biographie de Shakespeare (surtout tirées des recherches d'E. K. Chambers ${ }^{40}$ )

34 « [Grig] howls. He howls. He howls. He howls. He howls. He howls. He howls. (...) This is the scene of the seven howls. " The Crime of the Twenty First Century, Scene Ten, op. cit., p. 269.

35 Edward Bond, Bingo, « scene of money and death », in Plays 3, Londres, Methuen, 1987.

$3^{6}$ Respectivement mise en scène de Jane Howell et John Dove, Northcott Theatre, Exeter, 14 novembre 1973 et mise en scène de Howard Davies, Other Place, Stratford Upon Avon, 10 novembre 1976.

37 Mise en scène d'Angus Jackson, créée à Chichester, 26 avril 2010.

38 Création au Gymnase du Lycée Aubanel, 13 juillet 1994. Bingo scènes d'argent et de mort, Paris, L'Arche, 1994.

39 The Fool, Scenes of Bread and Love in Edward Bond, Plays 3, op. cit., création : mise en scène de Peter Gill, Royal Court Theatre, Londres, 18 novembre 1975.

$4^{\circ}$ Manifestement en particulier les documents commentés du second volume de son William Shakespeare, A Study of Facts and Problem, Oxford, Clarenton Press, 1930 (nous nous référons ici à l'édition de 1961), mais probablement aussi de Samuel Schoenbaum, Shakespeare's Lives, Oxford, Clarendon Press, 1970, qui venait alors de paraître. Bond reprend les noms de lieux, (en particulier la propriété de «New Place » (voir Chambers, p. 95-99) et de ses partenaires financiers, en particulier Thomas Combe (ibid. p. 127-141), farouche partisan de l'instauration des enclosures, qui dans la pièce lève une milice pour réprimer les émeutes paysannes dans le sang - et à qui, dans la réalité, Shakespeare céda son épée dans son testament (ibid., p. 172). 
pour dresser son portait en propriétaire terrien dans sa retraite de Stratford des dernières années - mais il donne, on s'en doute, une vision très personnelle de cette supposée période de « ease, retirement and conversation of his friends ${ }^{41} »$. On y voit Shakespeare, dans le but de garantir sa sécurité financière, passer un accord avec des propriétaires voisins entérinant l'établissement des enclosures dans la région $4^{2}$, qui, il le sait, spolieront et affameront la majorité des paysans, avec lesquels on le voit par ailleurs entretenir longtemps des rapports mutuels de sympathie et de confiance 43 . Il assistera ensuite passivement, comme un spectateur de l'une de ses propres pièces, aux effets humains et sociaux immédiats de cette politique : supplice d'une jeune femme hors-la-loi, vagabonde et prostituée, éclatement de la communauté locale, radicalisation idéologique autour des sectes puritaines (dont les membres, à la fin de la pièce, s'exileront pour les colonies en Amérique 44), jusqu'à un meurtrier soulèvement populaire.

La pièce entend mettre les positions humanistes des pièces de Shakespeare à l'épreuve de la réalité de la vie humaine, sociale, et ce, directement dans la sienne - il s'agit évidemment par-là de critiquer le hiatus essentiel entre les fondements moraux de la société et ses fonctionnements pratiques. Le personnage Shakespeare, du fait de la valeur implicite de ses pièces, incarne une balise morale, mais, placé dans sa situation historique, il apparaît vite complice de la violence que déclenche autour de lui le mouvement des enclosures (incarnation de l'exploitation capitaliste des hommes et des ressources) et il se révèle n'être ainsi pas du tout à la hauteur de son œuvre. Reformulant en termes moraux l'affirmation de Samuel Schoenbaum, selon laquelle, les documents comptables des archives de Stratford - notoirement les

${ }^{41}$ Selon la formule de Nicholas Rowe dans l'introduction de son édition des Works of William Shakespeare, Londres, Jacob Tonson, 1709, p. xxxv.

42 Voir l'enquête haletante et minutieuse sur les méthodes et les actes des propriétaires terriens autour de Shakespeare in Chambers, op. cit., p. 141-152. Bond reprend les termes de l'accord d'indemnisation effectivement passé par Shakespeare en octobre 1614 avec le steward du Lord Chancellor Ellesmere, en cas de pertes éventuelles dues à l'instauration des enclosures. Voir S. Shoenbaum, William Shakespeare : a Documentary Life, Oxford, Clarendon Press, 1975, p. 231.

43 Allusion possible aux « tenn poundes » laissées par Shakespeare dans son testament aux « Poore of Stratford » (cité in Chambers, op. cit., p. 172).

${ }^{44}$ La convergence avec les thèses d'Adolphus William Ward sur les liens entre l'œuvre de Shakespeare et la colonisation de la Virginie ( "Shakespeare and the Makers of Virginia », in The Annual Shakespeare Lecture, Londres, British Academy \& Oxford University Press, 1919) est probablement indirecte, sinon fortuite. Bond affirme cependant s'être inspiré de Richard Nixon pour composer le personnage du leader des Puritains. 
seules sources matérielles primaires de ses biographies - montrent en Shakespeare « a man concerned with money, real estate, and social position [... whose] prosaic interests [...] would alienate those [of his partisans] nurtured on the romantic idea of the poet 45 », Bond entend ainsi montrer

a contradiction between what Shakespeare said as a dramatist and what he did as a property owner. That isn't Lear : Lear goes out on the heath and rages against the injustice of society and the cruelty of god. It is like a split between Lear and Shakespeare ${ }^{46}$.

Le fait est que Shakespeare n'apparaît jamais durant la pièce comme un écrivain aux yeux de sa communauté, mais seulement comme un riche bourgeois - à qui on prête des activités à Londres, importantes mais abstraites. Une savoureuse scène, inspirée d'une anecdote fameuse ${ }^{47}$, le montre dans une taverne en compagnie d'un Ben Jonson, provocateur mais pétri d'admiration ${ }^{48}$, venu lui rendre visite pour tenter de savoir s'il écrit toujours et à qui il refuse obstinément de répondre sur ce sujet.

La tension entre ce qu'on attend de Shakespeare et ce qu'on voit de lui dans la pièce est à son comble dans la scène suivante, courte et picturale ${ }^{49}$. Rentrant ivre chez lui ${ }^{50}$, Shakespeare se retrouve dans un " open space, flat, white, crisp, empty. The fields, paths, roads, bushes and trees are covered with smooth clean snow ${ }^{51}$. " Plutôt que de se hâter de rentrer chez lui tant qu'il peut encore tenir debout, il préfère s'asseoir sur ce tapis de neige intact et infini et entreprend alors un long monologue méditatif. Au terme de cette scène, Shakespeare sera rétabli comme auteur et trouvera le moyen d'être dans sa vie moralement conséquent avec le discours de son œuvre. Nous allons

45 Shoenbaum, op. cit., p. 16.

46 Entretiens avec David Tuaillon, op. cit.

47 Bingo, Scene Four, op. cit., p. 43-53. Bond exploite ici la fameuse allusion du journal du Révérend Ward (qui consigna la mémoire des voisins de Shakespeare à Stratford cinquante ans après sa mort) à un " merry meeting » entre Shakespeare, Michael Drayton, et Ben Jonson, qui aurait mal tourné (cité in Chambers, op. cit., p. 250.).

48 Son argumentaire ambivalent est très probablement inspiré des commentaires peu amènes de Jonson sur Shakespeare rapportés par Chambers, op. cit., p. 211.

49 Bingo, Scene Five, op. cit., p. 53-58.

$5^{\circ}$ Bond poursuit la légende du « merry meeting » : s’il a retranché un convive, et que la rencontre qu'il met en scène n'a rien de " joyeuse », Shakespeare a bel et bien trop bu ce qui le rendra malade : " and it seems drank too hard, for Shakespear died of a feavour there contracted... ", in Chambers, op. cit.

${ }^{51}$ Bingo, op. cit., p. 53 . 
nous arrêter à présent sur cette scène afin d'observer les moyens esthétiques qu'elle se donne pour accomplir cette opération.

Premier mouvement : rendre à ce Shakespeare bon bourgeois son statut d'écrivain qu'il a perdu dans sa société. Ceci est d'abord visuellement conditionné par la métaphore claire et délicate d'une feuille de papier encore vierge, que propose cette grande étendue de neige. Elle attend tacitement que l'écrivain, comme il avait su le faire avec des mots dans son œuvre, inscrive par sa propre personne les actes qui donneront le sens moral de sa vie, du monde. Isolé dans cette blancheur abstraite, muette et inerte, Shakespeare semble comme convoqué : «Instead of sitting at his desk and writing on it, he is the ink on the paper: his life, with all its determinant events, is now writing itself on the paper ${ }^{52}$. »

À l'intérieur de cette image, la réappropriation par Shakespeare de son statut d'écrivain en tant que tel s'accomplit par un retour à son œuvre, très matériel parce qu'il a lieu dans la matière même du théâtre : le langage. Seul (et ivre), Shakespeare laisse libre cours à une interrogation sur son existence et la coule en un monologue prolifique en images et en questions qui retrouve insensiblement une rhétorique relevant des soliloques de ses pièces. Toute sa vie défile en vanités cosmiques, oxymorons précieux, visions naturalistes : de sa jeunesse passée, dont la franchise a été effacée par ses compromissions postérieures, à sa vieillesse, sèche et brève qui ne parvient pas à être sereine. Contemplant la neige, Shakespeare se dit ainsi que le jeune poète qu'il a été aurait pris un bâton pour y écrire une chanson, comme « la main d'un enfant fouille dans la barbe d'un vieillard ». Cette image est prise dans un grand développement de son imagination. Il voit d'abord la neige du matin, que le soleil « aurait fondu en mortalité » 53 , comme un signe de la mort du vieil homme, ce qui permet à l'image de se développer encore :

and in the morning the old man dies, goes, taking the curls from the child's fingers into the grave, and the child laughs and plays under the dead man's window. New games. Now I'm old. Where is the child to touch me and lead me to the grave 54 ?

${ }^{52}$ Entretiens avec David Tuaillon, op. cit.

53 Traduction de J. Hankins, op. cit. p.72. «In the morning the sun would melt it into mortality. A child's hand fumbling in an old man's beard »Bingo, op. cit., p. 56 .

54 Ibid. 
Sa parole, introspective, et qui avance en prenant appui, en pleine confiance, sur son imagination, l'amène enfin à énoncer la conclusion morale de toute la pièce: au bout de sa vision de cette dernière neige fragile et qui disparaîtra bientôt, dans laquelle il s'est reconnu lui-même au seuil de sa mort, nostalgique d'une vérité que seule l'innocence de l'enfance pourrait lui accorder, il s'aperçoit que la poignée de neige qu'il tient dans sa main déjà froide ne fond pas et proclame :

Every writer writes in other men's blood. [...] But only a god or a devil can write in other men's blood and not ask why they spilt it and what it costs. Not this hand, that's always melted snow 55 .

Shakespeare énonce ici enfin la vérité de sa situation et il y parvient précisément parce qu'il le fait en termes poétiques, c'est-àdire comme une expérience qui s'élucide dynamiquement en images et dans une forme instinctivement théâtrale (le monologue imagé au présent), qui, dans ses pièces, était celle par laquelle ses personnages arrivaient à leur propre vérité. Il rejoint ainsi les interrogations de ses personnages, sur leur humanité et le sens de leur vie dans le monde, en particulier ses rois déchus : il retrouve, dans sa vie, la rage du roi Lear dans la lande :

Thou wert better in a grave than to answer with thy uncover'd body. Is man no more than this? [...] Unaccommodated man is no more but such a poor bare, forked animal as thou art 56 .

Cette tirade rappelle également le désenchantement rétrospectif de Richard II :

Thoughts tending to ambition, they do plot Unlikely wonders : how these vain weak nails May tear a passage through the flinty ribs

Of this hard world, my ragged prison walls, And, for they cannot, die in their own pride. [...] But whate'er I be,

Nor I nor any man that but man is

With nothing shall be pleas'd, till he he eas'd

With being nothing 57 .

55 Ibid., p. 57.

${ }^{5}$ King Lear, III.iv.94-101.

57 The Tragedy of King Richard the Second, v.v.19-23 et 39-42. 
Le personnage Shakespeare reconnaît sa responsabilité dans ses actes, comme Macbeth, ce qui lui permet de faire face à leurs conséquences :

I have liv'd long enough. My way of life

Is fall'n into the sere, the yellow leaf,

And that which should accompany old age,

As honour, love, obedience, troops of friends,

I must not look to have, but in their stead,

Curses, not loud but deep, mouth-honour, breath

Which the poor heart would fain deny and dare not 58 .

Pour être tout à fait juste avec le Shakespeare de Bond, il faut préciser qu'il a déjà prononcé dans la pièce un tel soliloque. L'aprèsmidi avant sa dérive nocturne dans la neige, on l'a vu veiller le cadavre de la Jeune Femme, suppliciée sur un gibet au pied duquel se retrouvent un couple de paysans bruegéliens en pique-nique et un groupe de puritains en prière ${ }^{59}$. Shakespeare ne dit rien de la situation présente, mais il se lance dans une tirade terrible sur la cruauté des combats d'ours qui se tenait notoirement à côté de son théâtre ${ }^{60}$, métaphore évidente de la macabre scène à laquelle il assiste. Ses images sont vigoureuses, mais son langage est brut, brutal, réduit par la colère à des lambeaux de phrases et parfois à l'onomatopée :

In London, they blinded a bear. Called Harry Junks. The sports was to bait it with whips. Slash, slash. It couldn't see but it could hear. It grabbed the whips. Caught some of them. Broke them. Slashed back at the men. Slash, slash. The men stood round in a circle slashing at it. It was blind but they still chained it to the ground. Slash, slash. Then they sent an ape on a horse. A thin hairy man or a child. You could see the pale skin under its arm when it jumped. Its teeth. The dogs tore it to pieces. The crowd howled. London. ${ }^{61}$

On relèvera au passage que cette image est un emprunt (conscient ?) à Macbeth, où le personnage, acculé par ses ennemis, se

\footnotetext{
58 Macbeth, v.iii.23-30.

59 Bingo, Scene Three, op. cit., p. 34. Bond décrit explicitement cette image d'après une eau-forte de Rembrandt.

${ }^{60} \mathrm{La}$ proximité du Globe avec une salle de combat d'ours est attestée par le plan de Cornelius Visscher. Bond imagine que les représentations des pièces de Shakespeare auraient ressemblé à « un orchestre de chambre jouant à côté d'une foule de supporters de football. » (Entretiens avec David Tuaillon, op. cit.).

${ }^{61}$ Bingo, Scene Three, op. cit., p. 39.
} 
voit comme un ours attaché à un poteau ${ }^{62}$. Shakespeare trahit ainsi une conscience sincèrement meurtrie, mais il est encore incapable d'engager sa parole et sa vision d'écrivain dans ce qui se passe autour de lui. La scène lui permet de voir et de décrire, mais ses métaphores ne font qu'aliéner son expérience, et son art reste encore un seul moyen de transfert de la réalité vers l'imaginaire.

Pour clore la scène, Shakespeare raconte avoir vu la veille, alors qu'il marchait à travers la campagne (précisément pour fuir l'exécution de la Jeune Femme) un cygne, glissant sur l'Avon, et qui significativement s'éloignait de lui, jusqu'à disparaitre sans qu'il sache où ${ }^{63}$, image limpide, à travers ce symbole spéculaire, de l'absence à luimême croissante de l'écrivain. La scène suivante s'ouvrira par la description par Ben Jonson de l'incendie du Globe ${ }^{64}$ qui complète dans le réel cette vision de disparition.

Shakespeare aura ainsi assisté à la fuite de son propre symbole, appris la destruction physique du lieu du déploiement de son art et de sa gloire, subi la contestation de son rival et admirateur avant, enfin, de se retrouver au milieu de cet espace vierge et vide où tout est effacé où donc tout est de nouveau possible -, qui a la forme du lieu même de l'écrivain, la feuille blanche. C'est finalement là, à l'endroit où au terme de ce parcours se rejoignent son œuvre et le monde, que Shakespeare peut de nouveau regarder sa situation en écrivain, pour découvrir que ses pièces valent mieux que lui et qu'elles le somment d'accréditer leur discours par ses actes. Son corps, sa vie même, doivent désormais écrire comme sa plume, mais cette fois dans le réel et transformer, dit Bond, le "privilège » de l'écrivain en sa "responsabilité 65 ». Ces images constituent autant de chocs qui, s'accumulant, le poussent à cesser de garder sa conscience (et son art) pour lui et à l'engager dans le monde.

Une fois acceptée dans son principe la confrontation avec la réalité, encore lui faut-il s'accomplir concrètement. La scène

\footnotetext{
62 « MACBETH. They have tied me to a stake, I cannot fly, / But bear-like I must fight the course. » Macbeth, v.vii.1-2. Comme nous le signale Charlotte Coffin, cette image se retrouve dans plusieurs autres pièces de Shakespeare dont King Lear III.vii.53 («GlOUCESTER. I am tied to the stake and must stand the course ») ou Twelfth Night, III.i.120 ( OLIVIA. Have you not set mine honour at the stake »).

63 Bingo, Scene Three, op. cit., p. 41-42.

64 Ibid., Scene Four, p. 43. Bond a retardé la catastrophe de trois ans pour les besoins de la fiction, puisque le théâtre a en réalité brûlé en 1613 .

65 Entretiens avec David Tuaillon, op. cit.
} 
l'entreprend en exploitant une autre dimension de la métaphore visuelle : l'image d'idéal véhiculée par la blancheur immaculée de la surface neigeuse. Idéal de la création artistique souveraine, sublime, en gloire, retranchée dans le domaine pur de son immanence et qui ne doit rien au monde ni aux hommes qui l'ont produite ; idéal de l'artiste justifié par son seul génie - et qui d'autre que Shakespeare peut incarner cette icône du sublime sur une scène britannique ? Mais aussi idéal de pureté d'une morale si forte et si juste dans ses principes qu'elle refuse de se souiller au contact des réalités complexes et contradictoires du monde tel qu'il est - ce qui constitue le fond de la fracture tragique entre le personnage Shakespeare et son œuvre censée le représenter.

La scène envoie trois signaux à Shakespeare pour lui rappeler la présence de la réalité - laquelle, en effet, suit son cours, en arrière-plan de sa méditation intime, tout près, mais qu'il ne voit pas. Il y a une brève irruption de sa fille Judith, qui l'accable de reproches avant de l'abandonner à lui-même dans la neige. C'est elle qui, dans la pièce, veille aux intérêts financiers de la maison Shakespeare - et attend l'héritage avec avidité (une grande partie de la pièce évoque des querelles d'argent avec elle ${ }^{66}$ ). Judith constitue un rappel de la vénalité et de la haine qui corrompent les rapports humains. Elle a été précédée dans la scène par le jardinier de Shakespeare. C'est un vieil homme retombé en enfance à la suite d'une blessure de guerre, figure de l'innocence intacte - la scène le montre jouant dans la neige. S'ensuit enfin le passage, fugace, dans la brume, d'incertaines "silhouettes sombres ${ }^{67}$ » qui passent plusieurs fois en courant et qui se révèleront être des hommes au combat. Durant ce combat, qu'on devinera seulement à l'éclair d'un coup de feu au loin, un innocent sera tué, le

\footnotetext{
66 Bond développe ici aussi, avec une certaine ironie, les interprétations et les spéculations sur le testament de Shakespeare, semble-t-il rédigé à la hâte, sur son lit de mort et dont les legs à sa fille Judith (et la méfiance vis-à-vis de l'époux de celle-ci, Thomas Quincey, dont il semble témoigner, voir Chambers, op. cit., p. 175-176) est un des aspects les plus commentés par les biographes. Cependant, là où ces derniers entretiennent des soupçons sur la vénalité du gendre, le Shakespeare de la pièce reproche avec véhémence à sa fille sa corruption, s'en rendant lui-même responsable (Bingo, op. cit., p. 56). Bond, enfin, ajoute encore à la spéculation en montrant Judith qui extorque à Shakespeare mourant son testament (Scene 6, p. 60-61) - et laisse même imaginer qu'elle aurait pu le falsifier. La fin de la pièce la montre en train de remuer la pièce de fond en comble par-dessus le corps mort de son père, à la recherche d'un autre testament secret (Scene 6, p. 66).

67 «Four or five dark figures pass quickly over the top of the stage »Bingo, scene 5, p. 54 \& sqq.
} 
vieux jardinier de Shakespeare justement, atteint par une balle perdue tirée par son propre fils. Ce dernier meurtre, comme un retour forcé au réel, constitue le dernier élément qui pousse Shakespeare à sa décision.

La réponse du personnage Shakespeare à cette mise en demeure par son œuvre même, de s'engager dans le monde, entendra prouver la possibilité d'un geste moral malgré la corruption du monde. Son geste sera paradoxal et radical et, comme souvent dans les catastrophes tragiques des pièces de Bond, énigmatique - et en rien orthodoxe. Et nous n'en dirons rien ici afin de vous laisser le plaisir de la découverte en lisant la pièce ou en assistant à sa représentation. Il importe bien davantage de souligner que Shakespeare trouve la possibilité d'accomplir un geste moral en redevenant écrivain, au milieu de cette superbe métaphore visuelle d'une page de neige, et en interrogeant le statut véritable de l'écrivain dans le monde. Il affirme par-là que la seule véritable légitimité de l'art tient à son engagement dans la réalité.

C'était bien là la leçon reçue par le petit Bond découvrant sa propre vie dans Macbeth.

David TUAILLON 